\title{
Violência sexual contra mulheres em idade fértil na região norte do Brasil
}

\author{
Sexual violence against women of fertile age in the north region of Brazil
}

Violencia sexual contra las mujeres de edade fertil en la región norte de el Brasil

Kátia Fernanda Alves Moreira ${ }^{1}$, Bianca Oyola Bicalho², Tânia Leal Moreira ${ }^{3}$.

\begin{abstract}
RESUMO
Objetivo: Descrever o perfil das mulheres que sofreram violência sexual, bem como identificar as características do agressor e da violência sexual contra mulheres em idade reprodutiva. Métodos: Estudo quantitativo, descritivo, transversal realizado por meio de um levantamento de dados das variáveis no Sistema de Informação de Agravos de Notificação sobre a violência sexual ocorrida nas mulheres em idade fértil. Os dados foram analisados por meio de estatística descritiva no programa Epi InfoTM, versão 13.0. Resultados: No período estudado, houve 327 casos de violência sexual, sendo a maioria entre 10 a 19 anos $(83,18 \%)$, parda $(54,74 \%)$, solteiras $(77,06 \%), \leq 8$ anos de estudo $(49,85 \%)$. A agressão ocorreu no período noturno $(23,86 \%)$, na residência das vítimas $(59,33 \%)$, por conhecidos $(81,04 \%)$. Se tratando do atendimento oferecido $72,17 \%$ foi imediato nos serviços de saúde, com prescrição de profilaxia para Infecções Sexualmente Transmissíveis (45,87\%). Conclusão: A violência ocorre em todas as faixas etárias, independente da classe social, escolaridade ou estado civil, trazendo graves consequências a saúde biopsicossocial destas mulheres. Tais fatos justificam a discussão deste tema, subsidiando a implementação de políticas públicas de enfrentamento a violência e o estabelecimento de uma rede integral de atenção às mulheres a fim de minimizar os agravos.
\end{abstract}

Palavras-chave: Violência sexual, Violência contra a mulher, Estupro, Saúde pública.

\begin{abstract}
Objective: To describe the profile of women who suffered sexual violence, as well as to identify the characteristics of the aggressor and sexual violence against women of reproductive age. Methods: A quantitative, descriptive, cross-sectional study conducted through a survey of variables in the Notification Disease Information System about sexual violence in women of childbearing age. Data were analyzed using descriptive statistics using Epi InfoTM software, version 13.0. Results: During the study period, there were 327 cases of sexual violence, most of them between 10 and 19 years old $(83.18 \%)$, brown $(54.74 \%)$, single $(77.06 \%), \leq 8$ years of study $(49.85 \%)$. The aggression occurred at night $(23.86 \%)$, at the victims' residence (59.33\%), by acquaintances (81.04\%). Regarding the care offered $72.17 \%$ was immediate in health services, with prescription of prophylaxis for sexually transmitted infections (45.87\%). Conclusion: Violence occurs in all age groups, regardless of social class, education or marital status, bringing serious consequences to the biopsychosocial health of these women. These facts justify the discussion of this theme, supporting the implementation of public policies to confront violence and the establishment of an integral network of care for women in order to minimize the problems.
\end{abstract}

Keywords: Sexual violence, Violence against women, Rape, Public health.

${ }^{1}$ Fundação Universidade Federal de Rondônia Brasileira (UNIR), Porto Velho-RO.

2 Secretaria de Estado da Saúde de Rondônia (SESAU), Cacoal-RO. *E-mail: biancaylbicalho@gmail.com

${ }^{3}$ Secretaria Municipal de Saúde (SEMSAU), Estância Turística de Ouro Preto do Oeste-RO. 


\section{RESUMEN}

Objetivo: Escribir el perfil de las mujeres que sufrieron violencia sexual, así como identificar las características del agresor y la violencia sexual contra las mujeres en edad reproductiva. Métodos: Un estudio cuantitativo, descriptivo, transversal realizado a través de una encuesta de variables en el Sistema de Información de Enfermedades de Notificación sobre la violencia sexual en mujeres en edad fértil. Los datos se analizaron mediante estadísticas descriptivas con el software Epi InfoTM, versión 13.0. Resultados: Durante el período de estudio, hubo 327 casos de violencia sexual, la mayoría de ellos entre 10 y 19 años (83.18\%), marrón (54.74\%), soltero (77.06\%), $\leq 8$ años de estudio (49,85\%). La agresión ocurrió en la noche $(23.86 \%)$, en la residencia de las víctimas (59.33\%), por conocidos (81.04\%). En cuanto a la atención ofrecida, el $72,17 \%$ fue inmediato en los servicios de salud, con prescripción de profilaxis para las infecciones de transmisión sexual $(45,87 \%)$. Conclusión: La violencia ocurre en todos los grupos de edad, independientemente de la clase social, la educación o el estado civil, lo que trae graves consecuencias para la salud biopsicosocial de estas mujeres. Estos hechos justifican la discusión de este tema, apoyando la implementación de políticas públicas para enfrentar la violencia y el establecimiento de una red integral de atención a las mujeres para minimizar los problemas.

Palabras clave: Violencia sexual, Violencia contra la mujer, Violación, Salud pública.

\section{INTRODUÇÃO}

No Brasil, a idade fértil da mulher é considerada na faixa etária de 10 a 49 anos, essa definição foi realizada por meio de estudos de registros vitais e de procedimentos médicos que evidenciaram que nessa fase as mulheres estão expostas a riscos relacionados à vida sexual e reprodutiva (REZENDE FILHO J; MONTENEGRO CAB, 2014).

A violência sexual assim como a violência praticada pelo parceiro íntimo afeta uma grande parte da população sendo mulheres a maioria, que vivencia diretamente essas violências e a maioria que as perpetram, homens. A violência traz consigo as questões de gênero evidenciando o poder do homem sobre as mulheres, tornando-as objeto do seu poder, interferindo em sua autonomia como sujeito e desrespeitando os seus direitos (OLIVEIRA EM, 2007).

A violência contra a mulher é um problema de saúde pública com proporções epidêmicas no Brasil, embora sua magnitude ainda seja em grande parte invisível. Vale salientar que este problema não pode ser tratado de forma individualizada, visto que permeia toda a sociedade em seus diferentes segmentos. Para se prevenir e enfrentar de forma eficaz a violência contra a mulher, se faz necessário agir diretamente na redução das desigualdades de gênero, o que requer um trabalho integrado dos diferentes setores da sociedade, visando garantir que todas as mulheres independentes da faixa etária possam usufruir do direito básico de viver sem violência (GARCIA LP, 2016).

A violência sexual é uma brutal violação dos direitos, resultando em diversas consequências à mulher, como por exemplo, a morte e a incapacidade. Portanto, é um fenômeno com alta magnitude e vulnerabilidade constituindo-se não só um problema social, mas de saúde (GOMES VR, et al., 2014; ACOSTA DF, et al., 2015). Este tipo de violência bloqueia a autonomia da mulher e consequentemente 0 seu potencial como pessoa e membro da sociedade (AGUIAR JRV, et al., 2014).

Os dados sobre violência sexual ainda são insuficientes e fragmentados pelos altos índices de subnotificação, principalmente na região Norte, na qual houve nos últimos anos um aumento do número de mulheres vítimas de violência. Um dos motivos para a subnotificação da violência sexual é a dificuldade da sociedade em lidar com essa questão nos diversos setores, como o judiciário, segurança, educação e saúde (GOMES VR, et al., 2014).

Portanto, trabalhar essa temática é relevante pelo fato de acompanhar os índices de violência em Porto Velho e região após a construção das usinas hidrelétricas na capital, período que foi marcado por uma alta atividade de migração, ocasionando choques culturais, mudanças na economia, entre outros impactos. 
É importante destacar que o estudo permite a análise dos dados inseridos no sistema e com a divulgação dos resultados existe a possibilidade de sensibilização dos profissionais e, consequentemente, melhoria na qualidade do preenchimento do instrumento de notificação de violências.

Portanto, o objetivo do artigo é traçar o perfil da violência sexual contra a mulher residente do município de Porto Velho-RO, a fim de conhecer os dados epidemiológicos e provocar novas discussões, visando contribuir para o estabelecimento de políticas públicas de prevenção à violência e a maior qualidade no atendimento a estas vítimas.

\section{MÉTODOS}

Estudo quantitativo, descritivo, transversal realizado por meio de um levantamento de dados das variáveis no Sistema de Informação de Agravos de Notificação (SINAN) sobre a violência sexual ocorrida nas mulheres em idade fértil no período de janeiro de 2010 a dezembro de 2015, disponibilizados pela Secretaria de Estado da Saúde (SESAU) de Rondônia.

A população do estudo foi composta por todas as mulheres em idade fértil que sofreram violência sexual, residentes no município de Porto Velho, informados e registrados no SINAN. Os critérios de exclusão foram mulheres pré-púberes, com idade inferior a 10 anos e mulheres acima de 49 anos.

Para atender ao objetivo proposto, foram levantadas as seguintes variáveis: sociodemográficas (idade, raça/cor da pele, estado civil, escolaridade); relacionadas à violência sexual (horário do evento, local de ocorrência, relação com o agressor, outros episódios de violência sexual, suspeita de uso de álcool, circunstância da agressão, agressão sexual, zona de ocorrência).

E por fim, as informações relacionadas ao atendimento: classificação do atendimento, profilaxia para Infecções Sexualmente Transmissíveis (IST), Vírus da Imunodeficiência Humana (HIV), hepatite B, coleta de sangue, coleta de sêmen, coleta de secreção vaginal, contracepção de emergência e aborto previsto em lei.

Os dados coletados foram digitados em uma planilha no Excel, posteriormente armazenados em um banco de dados do programa Epi-Info versão 13.0 e analisados por meio de estatística descritiva. Este estudo atendeu aos aspectos éticos da Resolução 466/2012, conforme parecer 1.205.923 CEP/UNIR.

\section{RESULTADOS}

No período de 2010 a 2015 foram notificados 690 casos de violência contra à mulher no município de Porto Velho - RO, destes 327 casos foram de violência sexual, com maior frequência na faixa etária de 10 a 19 anos $(83,18 \%)$, mulheres autodeclaradas pardas $(54,74 \%)$, solteiras $(77,06 \%)$ e com baixa escolaridade $(49,85 \%)$. Ressalta-se o elevado percentual de registro "ignorado", nas variáveis escolaridade $(30,28 \%)$ e raça/ cor (13,15\%), respectivamente (Tabela 1). 
Tabela 1 - Distribuição das características sociodemográficas das mulheres em idade fértil vítimas de violência sexual no município de Porto Velho no período de 2010 a 2015.

\begin{tabular}{|c|c|c|}
\hline Variáveis sociodemográficas & $\mathrm{n}=327$ & $\%$ \\
\hline \multicolumn{3}{|l|}{ Idade (anos) } \\
\hline 10 a 19 & 272 & 83,18 \\
\hline 20 a 29 & 38 & 11,62 \\
\hline 30 a 39 & 8 & 2,45 \\
\hline 40 a 49 & 9 & 2,75 \\
\hline \multicolumn{3}{|l|}{ Raça/cor } \\
\hline Parda & 179 & 54,74 \\
\hline Branca & 69 & 21,10 \\
\hline Preta & 30 & 9,17 \\
\hline Amarela & 2 & 0,61 \\
\hline Indígena & 4 & 1,22 \\
\hline Ignorado & 43 & 13,15 \\
\hline \multicolumn{3}{|l|}{ Estado civil } \\
\hline Solteira & 252 & 77,06 \\
\hline Casada/União estável & 36 & 11,01 \\
\hline Separada & 4 & 1,22 \\
\hline Viúva & 1 & 0,31 \\
\hline Ignorado & 34 & 10,40 \\
\hline \multicolumn{3}{|l|}{ Escolaridade (anos) } \\
\hline$\leq 8$ & 163 & 49,85 \\
\hline 9 a 11 & 58 & 17,74 \\
\hline$\geq 12$ & 7 & 2,14 \\
\hline Ignorado & 99 & 30,28 \\
\hline
\end{tabular}

Fonte: SINAN - SESAU, 2016.

Em relação às características da violência, ocorria no período noturno $(23,86 \%)$, principalmente das 18:01 às $24: 00$ horas $(14,07 \%)$, na residência das vítimas $(59,33 \%)$, zona urbana $(36,09 \%)$. Algumas mulheres já haviam sofrido outros episódios de violência $(49,85 \%)$, o agressor não havia feito uso de bebidas alcoólicas $(48,01 \%)$ ou força física $(47,09 \%)$. Em relação ao tipo de agressão sexual praticada, predominou o coito vaginal $(42,20 \%)$, seguido pelo oral $(13,46 \%)$, porém o elevado percentual de registro "ignorado" em todos estes tipos de agressão (Tabela 2). 
Tabela 2 - Distribuição das mulheres em idade fértil vítimas de violência sexual em relação às características da violência no município de Porto Velho no período de 2010 a 2015

\begin{tabular}{|c|c|c|}
\hline Variáveis relacionadas à violência & $\mathrm{n}=\mathbf{3 2 7}$ & $\%$ \\
\hline \multicolumn{3}{|l|}{ Horário de ocorrência da agressão } \\
\hline $18: 01$ às $24: 00$ & 46 & 14,07 \\
\hline 00:01 às 07:00 & 32 & 9,79 \\
\hline 07:01 às 12:00 & 21 & 6,42 \\
\hline $12: 01$ às $18: 00$ & 28 & 8,56 \\
\hline Ignorado & 200 & 61,16 \\
\hline \multicolumn{3}{|l|}{ Local da ocorrência } \\
\hline Residência & 194 & 59,33 \\
\hline Rua & 42 & 12,84 \\
\hline Bar & 5 & 1,53 \\
\hline Escola & 4 & 1,22 \\
\hline Trabalho & 3 & 0,92 \\
\hline Outros & 35 & 10,70 \\
\hline Ignorado & 44 & 13,46 \\
\hline \multicolumn{3}{|l|}{ Outros episódios de violência sexual } \\
\hline Sim & 163 & 49,85 \\
\hline Não & 122 & 37,31 \\
\hline Ignorado & 42 & 12,84 \\
\hline \multicolumn{3}{|l|}{ Suspeita de uso de álcool } \\
\hline Não & 157 & 48,01 \\
\hline Sim & 82 & 25,08 \\
\hline Ignorado & 88 & 26,91 \\
\hline \multicolumn{3}{|l|}{ Agressão física } \\
\hline Não & 154 & 47,09 \\
\hline Sim & 125 & 38,23 \\
\hline Ignorado & 48 & 14,68 \\
\hline \multicolumn{3}{|l|}{ Tipo de agressão sexual } \\
\hline \multicolumn{3}{|l|}{ Coito vaginal } \\
\hline Sim & 138 & 42,20 \\
\hline Não & 7 & 2,14 \\
\hline Ignorado & 182 & 55,66 \\
\hline \multicolumn{3}{|l|}{ Coito oral } \\
\hline Não & 75 & 22,94 \\
\hline Sim & 44 & 13,46 \\
\hline Ignorado & 208 & 63,61 \\
\hline \multicolumn{3}{|l|}{ Coito anal } \\
\hline Não & 89 & 27,22 \\
\hline Sim & 24 & 7,34 \\
\hline Ignorado & 214 & 65,44 \\
\hline \multicolumn{3}{|l|}{ Mais de um tipo } \\
\hline Não & 212 & 64,83 \\
\hline Sim & 29 & 8,87 \\
\hline Ignorado & 86 & 26,30 \\
\hline \multicolumn{3}{|l|}{ Zona de ocorrência } \\
\hline Urbana & 256 & 78,29 \\
\hline Rural & 31 & 9,48 \\
\hline Ignorado & 40 & 12,23 \\
\hline
\end{tabular}

Fonte: SINAN - SESAU, 2016. 
A relação entre as vítimas de violência sexual com o agressor verificou-se que $81,04 \%$ dos agressores eram conhecidos pela vítima, categorizados principalmente como amigos $(26,30 \%)$ ou namorado $(22,63 \%)$ (Tabela 3).

Tabela 3 - Distribuição da relação entre o agressor e as mulheres em idade fértil vítimas de violência sexual no município de Porto Velho no período de 2010 a 2015.

\begin{tabular}{|c|c|c|}
\hline Relação com o agressor & $\mathrm{n}=327$ & $\%$ \\
\hline Conhecido & 265 & 81,04 \\
\hline Amigos & 86 & 26,30 \\
\hline Namorado & 74 & 22,63 \\
\hline Padrasto & 25 & 7,65 \\
\hline Pai & 18 & 5,50 \\
\hline Conjuge & 16 & 4,89 \\
\hline Ex-namorado & 6 & 1,83 \\
\hline Irmão & 4 & 1,22 \\
\hline Mãe & 3 & 0,92 \\
\hline Ex-conjuge & 3 & 0,92 \\
\hline Avô & 2 & 0,61 \\
\hline Pessoal com relação institucional & 2 & 0,61 \\
\hline Outros* & 26 & 7,95 \\
\hline Desconhecido & 62 & 18,96 \\
\hline
\end{tabular}

Legenda: *Cunhado, primo, vizinho, tio, professor, cuidador, patrão/chefe. Fonte: SINAN - SESAU, 2016.

No que diz respeito as condutas assistenciais prestadas as estas mulheres vítimas de violência sexual, a maioria foi imediato nos serviços de saúde $(72,17 \%)$, com prescrição de profilaxia para IST $(45,87 \%)$ e Hepatite B $(33,03 \%)$, bem como uso de antirretrovirais $(38,23 \%)$. Além disso, a maioria realizou coleta de sangue $(64,53 \%)$ ou de outros materiais, tais como secreção vaginal $(2,45 \%)$ ou sêmen $(0,92 \%)$. Entretanto, não houve prescrição de contracepção de emergência $(45,57 \%)$ dos casos e não realizaram aborto previsto em lei (66,06\%) (Tabela 4). 
Tabela 4 - Distribuição das características do atendimento as mulheres em idade fértil vítimas de violência sexual no município de Porto Velho no período de 2010 a 2015

\begin{tabular}{|c|c|c|}
\hline Variáveis relacionadas ao atendimento & $\mathrm{n}=327$ & $\%$ \\
\hline \multicolumn{3}{|l|}{ Classificação do atendimento } \\
\hline Imediato (até 5 dias) & 236 & 72,17 \\
\hline Tardio (após 5 dias) & 91 & 27,83 \\
\hline \multicolumn{3}{|l|}{ Profilaxia de IST } \\
\hline Sim & 150 & 45,87 \\
\hline Não & 118 & 36,09 \\
\hline lgnorado & 59 & 18,04 \\
\hline \multicolumn{3}{|l|}{ Profilaxia de HIV } \\
\hline Não & 140 & 42,81 \\
\hline Sim & 125 & 38,23 \\
\hline Ignorado & 62 & 18,96 \\
\hline \multicolumn{3}{|l|}{ Profilaxia Hepatite B } \\
\hline Não & 149 & 45,57 \\
\hline Sim & 108 & 33,03 \\
\hline Ignorado & 70 & 21,41 \\
\hline \multicolumn{3}{|l|}{ Coleta de sangue } \\
\hline Sim & 211 & 64,53 \\
\hline Não & 66 & 20,18 \\
\hline Ignorado & 50 & 15,29 \\
\hline \multicolumn{3}{|l|}{ Coleta de secreção vaginal } \\
\hline Não & 222 & 67,89 \\
\hline Sim & 8 & 2,45 \\
\hline Ignorado & 97 & 29,66 \\
\hline \multicolumn{3}{|l|}{ Contracepção de emergência } \\
\hline Não & 149 & 45,57 \\
\hline Sim & 95 & 29,05 \\
\hline Ignorado & 83 & 25,38 \\
\hline \multicolumn{3}{|l|}{ Aborto previsto em lei } \\
\hline Não & 216 & 66,06 \\
\hline Sim & 11 & 3,36 \\
\hline Ignorado & 100 & 30,58 \\
\hline \multicolumn{3}{|l|}{ Coleta de sêmen / n=236 } \\
\hline Não & 162 & 70,64 \\
\hline Sim & 2 & 0,92 \\
\hline Ignorado & 72 & 28,44 \\
\hline
\end{tabular}

Legenda: Infecção Sexualmente Transmissível (IST) / Vírus da Imunodeficiência Humana (HIV). Fonte: SINAN - SESAU, 2016.

\section{DISCUSSÃO}

Apesar de muitos fatores contribuírem para o aumento do número de mulheres que sofrem agressões, a violência contra as mulheres atinge todas as classes sociais, independente da sua cor/raça, escolaridade, estado civil, idade e em qualquer fase da vida da mulher (ACOSTA DF, et al., 2015).

Neste estudo ao ser analisados os dados sociodemográficos das mulheres vítimas de violência sexual, constatou-se que $83,18 \%$ tinham entre 10 a 19 anos. Estudo em São Paulo tiveram resultados semelhantes, 
em que a maioria das vítimas tinha $\leq 19$ anos (FACURI CO, et al., 2013). As adolescentes estão mais expostas a violência sexual, ou seja, são frequentemente vitimizadas não tendo maturidade de compreender a situação de agressão sofrida, devido a vulnerabilidade da própria idade (MIRANDA MIF, et al., 2014). Quanto à raça/cor autodeclarada prevaleceu a parda $(54,74 \%)$, até mesmo pela configuração da população do estado, o que diferencia de outras pesquisas realizadas em diferentes regiões do país, em que predominou a cor branca (ACOSTA DF, et al., 2013; FACURI CO, et al., 2013).

Quanto ao estado civil, $77,06 \%$ eram solteiras, resultados similares foram encontrados em um estudo no estado de São Paulo, em que $76,1 \%$ das mulheres vítimas de violência sexual também eram solteiras (FACURI CO, et al., 2013). Vale ressaltar que tal resultado pode ter sido influenciado devido à idade das vítimas que são adolescentes na sua maioria.

Nos estudos realizados no Rio Grande do Sul e no Paraná, a maioria das mulheres tinha baixa escolaridade, corroborando com essa pesquisa, em que 49,85\% tinham $\leq 8$ anos de estudos (ACOSTA DF, et al., 2013; TRIGUEIRO TH, et al., 2015).

Autores atribuem como fatores de enfrentamento e proteção às violências de gênero o acesso ao estudo e emprego, diminuindo em $60 \%$ a chance das mulheres de sofrerem violência sexual (PURI M, et al., 2012). Neste estudo, o horário de maior ocorrência foi o período noturno $(23,86 \%)$.

Tais achados corroboram com um estudo realizado em São Paulo em que o estupro ocorreu principalmente no final do dia e na madrugada correspondendo a $34,9 \%$ e $41,0 \%$ respectivamente (FACURI CO, et al., 2013).

Quanto ao local da agressão prevaleceu a residência das vítimas $(59,33 \%)$, sendo $81,04 \%$ dos agressores conhecidos das vítimas. Esses resultados são semelhantes aos encontrados em estudo realizado em Sergipe, em que predominou a violência praticada por agressores conhecidos $(84,8 \%)$ (SOUSA LC, et al., 2013).

Os crimes de natureza sexual são praticados no espaço de convivência da criança e do adolescente, por pessoas próximas e que desfrutam de certa confiança por parte das vítimas (MIRANDA MIF, et al., 2014), principalmente por homens (VELOSO MMX, et al., 2013).

Ainda hoje, a violência contra a mulher é tratada como um assunto restrito e cheio de conflitos, no qual o senso comum e os comentários vêm acompanhados de um preconceito, carregado de valores de gênero, que se perpetuam durante séculos, geração após geração. Romper essa linha de pensamento requer uma força da sociedade, em conjunto com a academia, na formação de profissionais mais acolhedores e humanos ao lidar com as especificidades das mulheres, particularmente as que são vítimas de violência.

Acresce-se a este fato que as situações de maior vulnerabilidade para a violência sexual sofrida por mulheres apresentam-se justamente dentro do círculo supostamente seguro do lar, da família e dos amigos. Tal circunstância contribui para a invisibilidade da violência tornando o crime mais difícil de ser denunciado e mantendo a impunidade dos agressores (ACOSTA DF, et al., 2015).

Dos casos analisados $49,85 \%$ das mulheres declararam ter sofrido outros episódios de violência, o mesmo ocorreu em um estudo realizado na região sul do Brasil em que $63 \%$ dos casos referiam agressões anteriores (ACOSTA DF, et al., 2013). A violência sexual possui o registro extremamente complicado, por se constituir em um evento em que existe uma marca registrada, um pacto de silêncio (SANTOS TMB, et al., 2014).

O fato de as vítimas serem meninas, na faixa etária que caracteriza a adolescência, é comum culpá-las pela agressão, por estarem em uma idade na qual despertam a feminilidade, devido ao aparecimento dos caracteres sexuais secundários (MIRANDA MIF, et al., 2014). Isso gera medo nas vítimas pelo receio em ser julgada pela família, amigos e conhecidos levando-as a internalizar seus sentimentos e percepções.

A ingestão do álcool contribui, mas não necessariamente é o único fator suficiente para a execução do ato violento (LINDNER SR, et al., 2015). Ou seja, ele representa um fator precipitante da violência, por seu efeito desinibidor da conduta dos agressores ou como um meio de minimizar a responsabilidade pelo 
comportamento (MOURA LBA, et al., 2009). Em um estudo realizado no município de Rio Grande-RS, verificou-se o uso de álcool por $82,6 \%$ dos indiciados nos inquéritos que continham esse registro (SILVA $C D$, et al., 2013). Em outro estudo no estado de Sergipe o uso de álcool e/ ou drogas pelos agressores estava presente em $25 \%$ dos casos (SOUSA LC, et al., 2013).

No entanto, a realidade de Porto Velho chama a atenção, visto que neste estudo $48,01 \%$ dos casos não havia suspeita de o agressor ter feito uso de bebida alcoólica, estando lúcidos no momento que perpetraram a violência. Na população de estudo, observou-se que os agressores não fizeram uso da força física em $47,09 \%$ das mulheres, como forma de intimidá-las.

Tais dados se distanciam de um estudo realizado no sul do país em que $63,4 \%$ das agressões sexuais foram acompanhadas de agressão física como forma de intimidação; nestas $43,9 \%$ houve força corporal e ameaça (ACOSTA DF, et al., 2015).

Dados do SINAN de 2011 reforçam que, em $56 \%$ dos casos, a força corporal ou o espancamento são os meios mais utilizados pelos agressores na violência contra a mulher (WAISELFISZ JJ, 2012). É preciso considerar o pequeno número de casos em que a violência sexual não é acompanhada de agressão física, mas de violência psicológica, como naqueles em que a pessoa se sente coagida recebendo ameaças ou até mesmo seduzidas o que contribui para o silêncio da vítima.

Acerca do tipo de agressão sexual praticada as principais categorias identificadas foram o coito vaginal com $42,20 \%$ dos casos, porém a informação ignorada nas notificações prevaleceu em todos os tipos. Os achados encontrados convergem com os de um estudo realizado em Teresina-PI em que o tipo de violência sexual praticada foi o sexo vaginal (39,0\%) (SOARES EMR, et al., 2016).

A violência sexual com penetração vaginal é a mais praticada contra as mulheres segundo estudos nacionais (FACURI CO, et al., 2013). O que chama atenção neste estudo é a porcentagem em que as vítimas sofreram penetração oral (13,46\%), anal $(7,34 \%)$ e mais de um tipo $(8,87 \%)$, demonstrando a severidade da agressão e aumento do risco de transmissão das ISTs e do HIV. Tal situação retrata a gravidade das violências e que o agressor utiliza de várias formas de agressão, algo provavelmente associado à cultura machista, heterossexista e patriarcal (ACOSTA DF, et al., 2015).

Violência sexual contra mulher é um ato hostil, agressivo, utilizado pelos agressores como meio de degradar, humilhar, aterrorizar e dominar, assumindo uma característica multifacetada. Os motivos do silêncio das mulheres acerca da violência sexual sofrida são diversos e o medo de represálias dificulta as estimativas da magnitude desse tipo de violência.

Verificou-se ao analisar os dados que $78,29 \%$ das mulheres residiam na zona urbana do município pesquisado. No entanto, os baixos índices de violência registrados por mulheres que vivem na zona rural, podem estar associados à dificuldade de acesso aos serviços de atenção às vítimas de violência, seja pela distância e/ ou situação econômica (FERRAZ MIR, et al., 2009).

Outro fator a se considerar é o processo de divisão do trabalho no meio rural, que ainda ocorre de forma sexista, contribuindo para a invisibilidade da identidade feminina, reforçando sua dependência ao homem. Tal situação contribui para a perpetuação da dominação masculina, inserindo a violência no espaço do silêncio e segredo dos lares (ARBOIT J, et al., 2015). Desta forma, os achados deste estudo, na qual a minoria das notificações era residente na zona rural deve ser motivo de preocupação e investigações (RAIMONDO ML, et al., 2013).

No que concerne ao atendimento nos serviços de saúde, $72,17 \%$ das vítimas tiveram atendimento imediato, ou seja, a maioria recebeu as medidas profiláticas em tempo hábil, resultando na maior eficácia do anticoncepcional de emergência e a quimioprofilaxia das IST (FACURI CO, et al., 2013). Como grande parte dos crimes de violência sexual ocorrem nesta fase reprodutiva da mulher, três situações são relevantes quando se pensa no tratamento, é o risco de gravidez, as IST e o HIV.

Em relação as prescrições de profilaxias, $45,87 \%$ das mulheres receberam para as IST, $38,23 \%$ receberam para uso de antirretrovirais, 33,03\% para profilaxia de Hepatite B, $64,53 \%$ das mulheres realizaram coleta de sangue e $2,45 \%$ secreção vaginal. 
A violência sexual pode ser um dos fatores que contribuem para que a população adolescente seja uma das mais infectadas com as IST, sendo importante que se institua rotinas para garantir imediatamente a realização dos exames, dentre outras condutas (BRASIL, 2012).

A coleta de sêmen foi realizada somente em $0,92 \%$ dos casos tal conduta é importante para a identificação do agressor e condenação do culpado posteriormente (SOUSA JM, et al., 2012). No entanto, o percentual está conforme o esperado, visto que não se trata de um procedimento obrigatório nos serviços que prestam atendimento a este público (BRASIL, 2012).

Já a prescrição de contracepção de emergência, a maioria das mulheres não recebeu, correspondendo a $45,57 \%$ dos casos, o que aumenta a chance de a vítima engravidar e realizar um aborto, como ocorreu em $3,36 \%$ das vítimas de violência sexual deste estudo, que optaram pelo aborto previsto em lei. Entre as consequências da violência sexual, a gravidez se destaca pela complexidade das reações psicológicas, sociais e biológicas. A gestação indesejada ou forçada é encarada como uma segunda violência, intolerável para muitas mulheres (BRASIL, 2012).

A violência contra as mulheres provoca lesões e incapacidade nas vítimas nas diferentes partes do mundo, sendo também um fator de risco a outros aspectos como o bem-estar materno-infantil, além das consequências sociais e econômicas (OPS, 2014).

É fundamental o reconhecimento dos serviços de saúde quanto ao impacto das desigualdades de gênero na vida e a saúde da população, para que assim possam intervir, de modo a prestar um cuidado integral e a garantia dos direitos das mulheres (TERRA MF e d'OLIVEIRA AFPL, 2015), uma vez que são esses serviços a porta de entrada na rede. Desse modo, estratégias como o mapeamento e o fortalecimento das redes locais, se fazem importantes para o enfrentamento desse tipo de violência na demanda dos atendimentos nos serviços de saúde nos diferentes níveis de complexidade (TREVISAN SB, et al., 2015).

Entre as limitações desse estudo, podemos apontar a influência da subnotificação, especialmente por se tratar de violência sexual. Além disto, verificou-se que a maioria das variáveis tinha um elevado percentual como "ignorado", o que interfere na qualidade do monitoramento das ações e na avaliação do sistema, além de ser um indicativo de que as informações, possivelmente, não foram priorizadas pelos profissionais da saúde. As variáveis merecem atenção no treinamento para o preenchimento da ficha de notificação de violência, haja vista seu potencial para auxiliar na mensuração das desigualdades sociais, acesso aos serviços de saúde e sua associação com a ocorrência da violência.

\section{CONCLUSÃO}

Esse estudo reafirma que a violência sexual ocorre em todas as faixas etárias, independente da classe social, escolaridade ou estado civil, trazendo graves consequências a saúde biopsicossocial destas mulheres. Tais fatos indicam que este tema continue sendo objeto de estudo e subsidie a implementação de políticas públicas de enfrentamento da violência sexual e suas implicações. Além do estabelecimento de uma rede integral de atenção às mulheres em idade fértil vítimas de violência em busca da recuperação nas relações interpessoais e no tratamento integrado dos agravos à saúde provenientes de tal violência, a fim de minimizar os danos ocasionados.

\section{REFERÊNCIAS}

1. ACOSTA DF, GOMES VLO, BARLEM ELD. Perfil das ocorrências policiais de violência contra a mulher. Revista Acta Paulista de Enfermagem, 2013; 26(6): 547-553.

2. ACOSTA DF, GOMES VLO, FONSECA AD, GOMES GC. Violencia contra a mulher por parceiro íntimo: (In)visibilidade do problema. Texto Contexto Enfermagem, 2015; 24(1): 121-7.

3. AGUIAR JRV, SILVA GME, MONTE NL. Estudo sobre a violência sexual em Serviço de Atendimento à Mulher. Revista Enfermagem UFPI, 2014; 3(4): 79-87.

4. ARBOIT J, COSTA MC, HIRT MC, PADOIN SMM, COLOMÉ ICS, SOARES JSF. Violência doméstica contra mulheres rurais: interfaces de gênero na concepção de agentes comunitários de saúde. Revista Ciencia, Cuidado e Saúde, 2015; 14(2): 1067-1074. 
5. BRASIL. Ministério da Saúde. Prevenção e tratamento dos agravos resultantes da violência sexual contra mulheres e adolescentes: norma técnica. Secretaria de Atenção à Saúde. Departamento de Ações Programáticas Estratégicas. - 3. ed. atual. Brasília: Ministério da Saúde. 2012; 124 p.

6. FACURI CO, FERNANDES, MAS, OLIVEIRA KD, ANDRADE TS, AZEVEDO RCS. Violência sexual: estudo descritivo sobre as vítimas e o atendimento em um serviço universitário de referência no Estado de São Paulo, Brasil. Caderno de Saúde Pública, 2013; 29(5): 889-898.

7. FERRAZ MIR, LABRONICI LM. Perfil da violência doméstica contra mulher em Guarapuava, Paraná. Revista Cogitare Enfermagem, 2009; 14(2): 261-268.

8. GARCIA LP. The invisible magnitude of violence against women. Revista Epidemiologia e Serviços de Saude, 2016; 25(3): 451-454.

9. GOMES VR, LIMA VLA, SILVA AF, SENA LX, SANTOS ACB. Violência contra a mulher na região norte: a versão da mídia impressa paraense. Revista LEVS/UNESP, 2014; ed 14: 113-128.

10. LINDNER SR, COELHO EBS, BOLSONI CC, ROJAS PF, BOING AF. Prevalência de violência física por parceiro íntimo em homens e mulheres de Florianópolis, Santa Catarina, Brasil: estudo de base populacional. Caderno de Saúde Pública, 2015; 31(4): 815-823.

11. OLIVEIRA EM. Fórum Violência sexual e saúde. Introdução. Caderno de Saúde Pública, 2007; 23(2): $455-458$.

12. REZENDE FILHO J, MONTENEGRO CAB. Rezende: obstetrícia fundamental. 13ª ed. Rio de Janeiro: Guanabara Koogan; 2014; 1801p.

13. MIRANDA MIF, SANTOS MAM, TOURINHO MBAC, WATANABE CV, MIGUEL VVR, SANTOS GDN. Violência sexual contra crianças e adolescentes em um município da Região Norte do Brasil. Revista da Sociedade Brasileira de Enfermagem Pediátrica, 2014; 14(2): 96-104.

14. MOURA LBA, GANDOLFI L, VASCONCELOS AMN, PRATESI R. Violências contra mulheres por parceiro íntimo em área urbana economicamente vulnerável, Brasília, DF. Revista de Saúde Pública, 2009; 43(6): 944-953.

15. OPS. Organización Panamericana de la Salud. Violencia contra las mujeres en América Latina y el Caribe: Análisis comparativo de datos poblacionales de 12 países. Washington, DC: OPS. 2014; 198p.

16. PURI M, FROST M, TAMANG J, LAMICHHANE P, SHAH I. The prevalence and determinants of sexual violence against young married women by husbands in rural Nepal. BMC Res Notes, 2012; 5: 291.

17. RAIMONDO ML, LABRONICI LM, LAROCCA LM. Retrospecto de ocorrências de violência contra a mulher um registradas em uma delegacia especial. Revista Cogitare Enfermagem, 2013; 18(1): 43-49.

18. SANTOS TMB, SOUZA TDA, ROCHA GSA, SILVA LMP. Analysis of the scientific production on the notification of violence against adolescents. Brazilian Journal in Health Promotion, 2014; 27(4): 560-567.

19. SILVA CD, GOMES VLO, ACOSTA DF, BARLEM ELD, FONSECA AD. Epidemiologia da violência contra a mulher: características do agressor e do ato violento. Revista de Enfermagem UFPE online, 2013; 7(1): 8-14.

20. SOARES EMR, SILVA NL, MATOS, MAS, ARAÚJO ETH, SILVA LR, LAGO EC. Perfil da violência sexual contra crianças e adolescentes. Revista Interdisciplinar, 2016; 9(1): 87-96.

21. SOUSA JM, QUEIROZ PRM. Coleta e preservação de vestígios biológicos para análises criminais por DNA. Ensaios e Ciência, 2012; 16(3): 99-115.

22. SOUSA LC, MACHADO LC, MIRANDA ACPT. Perfil sociodemográfico e epidemiológico das vítimas de violência sexual no Estado de Sergipe. Interfaces Científicas, Saúde e Ambiente, 2013; 1(3): 21-33.

23. TERRA MF, d'OLIVEIRA AFPL. Prevenção às DST/HIV/Aids para as mulheres em situação de violência doméstica de gênero: uma análise sobre a vulnerabilidade programática. Arquivos Médicos dos Hospitais e da Faculdade de Ciências Médicas Santa Casa São Paulo, 2015; 60(3): 117-121.

24. TREVISAN SB, LEAL SMC, FENSTERSEIFER LM. Characteristics of women in violence situation assisted at Jacobina Center. Journal Nurse UFPE on line, 2015; 9(9): 9197-206.

25. TRIGUEIRO TH, MERIGHI MAB, MEDEIROS ARP, RIBEIRO CEL, MATA NDS, JESUS MCP. Vítimas de violência sexual atendidas em um serviço de referência. Revista Cogitare Enfermagem, 2015; 20(2): 249-256.

26. VELOSO MMX, MAGALHÃES CMC, DELL'AGLIO DD, CABRAL IR, GOMES MM. Notificação da violência como estratégia de vigilância em saúde: perfil de uma metrópole do Brasil. Revista Ciência \& Saúde Coletiva, 2013; 18(5): 1263-1272.

27. WAISELFISZ JJ. Mapa da Violência 2012. Atualização: homicídios de mulheres. Brasília: CEBELA; FLASCO/Brasil. 2012; 27p. 